\title{
Transcatheter treatment of atherosclerotic lesions of the brain complicated by vascular dementia development
}

\author{
Ivan V. Maksimovich \\ Clinic of Cardiovascular Diseases named after Most Holy John Tobolsky, Moscow, Russia \\ Email: carvasc@yandex.ru
}

Received 26 June 2012; revised 30 July 2012; accepted 21 August 2012

\section{ABSTRACT}

The research focuses on the effectiveness of transluminal laser revascularization of the brain in the treatment of atherosclerotic lesions accompanied by vascular dementia development. 1125 patients aged from 29 to 81 (average age 75) suffering from various kinds of atherosclerotic lesions of cerebral vessels were examined during the research. The examination plan included: computed tomography of the brain (CT), magnetic resonance imaging (MRI), scintigraphy of the brain (SG), rheoencephalography (REG), cerebral multi-gated angiography (MUGA). 665 (59.11\%) patients suffered from diseases accompanied by the development of vascular dementia. To perform transcatheter treatment, 639 patients were selected: Group 1 (CDR-1) - 352 patients, Group 2 (CDR-2) - 184 patients, Group 3 (CDR-3) - 103 patients. To conduct revascularization of main intracranial arteries highenergy laser systems were used; for revascularization of the distal intracranial branches low-energy laser systems were used. The clinical outcome depended on the severity of dementia and the timing of the intervention. A good clinical outcome in Group 1 was obtained in 281 (79.82\%) cases, in Group 2 in $81(44.02 \%)$ cases, in Group 3 in $9(8.73 \%)$ cases. A satisfactory clinical outcome in Group 1 was obtained in 53 $(15.34 \%)$ cases, in Group 2 in $62(33.70 \%)$ cases, in Group 3 in $31(30.09 \%)$ cases. A relatively satisfactory clinical outcome in Group 1 was obtained in 17 $(4.83 \%)$ cases, in Group 2 in $41(22.28 \%)$ cases, in Group 3 in $63(61.16 \%)$ cases. No negative effect was observed after the intervention. Evaluating the data obtained it can be concluded that the method of transluminal laser revascularization of cerebral blood vessels is an effective one for the treatment of atherosclerotic lesions of the brain accompanied by dementia.

Keywords: Cerebrovascular Disease; Vascular Dementia; Cognitive Impairment; Stroke; Transcatheter Treatment; Laser Revascularization; Regeneration

\section{INTRODUCTION}

Cerebrovascular diseases occupy one of the leading positions on mortality among the population and are among the major causes of disability affecting middle-aged and senior citizens [1]. Lesions of the brain vascular system vary considerably and lead not only to motor and sensory disturbances but also contribute to the development of secondary neurodegenerative changes, mental disorders and dementia [2]. According to "The Seventh International Congress on Vascular Dementia”, the development of vascular dementia (VaD) is mostly associated with atherosclerotic lesions of some or other parts of cerebrovascular bed. Normally, the capillary bed plays the leading part in cerebral cells blood supply; pathologically, the capillary and collateral bed, which also has capillaries of its own, does. Large arteries are more likely to be the means of transport and only deliver blood to the capillaries [2,3]. According to some authors, the number of capillaries in a cubic centimeter of brain tissue makes 3 - 4 thousand [3]. Thus, the brain can be described as an organ with the highest level of blood supply.

Brain tissue is extremely sensitive to ischemia. The development of atherosclerotic lesions is a rather lengthy process. In some situations it is slower, usually widespread, affecting smaller blood vessels and causing transient attacks and micro lacunar strokes. It does not cause any hemodynamically significant stenoses or occlusions of larger arterial branches. A lesion of this kind causes chronic ischemia of the brain which in turn leads to neurodegenerative changes, cognitive disorders and progressing dementia [4-7].

In other situations, when small arterial branches in one of the pools are affected, the atherosclerotic process is faster, causing stenoses or occlusions of larger intracranial branches. This kind of disease progression leads to the development of larger strokes, also causing secondary neurodegenerative changes, the development of cognitive impairment and dementia [3,5,7,8].

Hyperlipidemia, hypertension, disorders of systemic hemodynamics and microembolia caused by cardiac rhythm disorders also play an important role in the deve- 
lopment of $\mathrm{VaD}$. They also contribute to the development of cerebral ischemia $[9,10]$.

The number of patients suffering from vascular dementia is constantly growing. According to many authors, $\mathrm{VaD}$ makes 20 and even $50 \%$ of all types of dementia and ranks second in frequency after Alzheimer's disease in different countries [11-13].

Conservative treatment of patients with $\mathrm{VaD}$ may be effective only for a certain period of time followed by further growing disorders in the cerebral hemodynamics which do not allow the improvement of blood circulation by therapeutic methods [14].

Reconstructive vascular surgery, well-established in the treatment of extracranial vessels, is rather difficult to be applied on patients with intracranial lesions due to the anatomical features [15]. Surgical interventions aimed at creation of extra-intracranial arterial anastomosis are not always effective, and debates on the feasibility of such surgical procedures have been going on for years [16].

Such interventions as balloon angioplasty and stenting of various kinds, which have also proved themselves quite effective in the extracranial parts of the brachiocephalic branches [15], reveal certain difficulties with small diameters of intracranial arterial branches. In this regard, there remains a need for the development of new transcatheter treatment methods.

\section{MATERIALS AND METODS}

\subsection{Subject of Research}

The whole research and all transcatheter interventions have been made with the approval of the Ethics Committee and with the consent of the examined and treated patients and their relatives.

1125 patients aged 29 to 81 (average age 75) suffering from various kinds of atherosclerotic lesions of cerebral vessels were examined during the research 842 (74.84\%) male patients and 283 (25.16\%) female patients.

\subsection{Examination of Patients}

The general research plan included: computed tomography of the brain (CT), magnetic resonance imaging (MRI), scintigraphy of the brain (SG), rheoencephalography (REG), cerebral multi-gated angiography (MUGA), laboratory diagnosis, and assessment of the severity of dementia, cognitive disorders and everyday life disorders.

$\mathrm{CT}$ and MRI of the brain were performed on apparatus "Somatom" (Siemens), "HiSpeed" (GE), "Tomoscan" (Philips), "Apetro Eterna” (Hitachi) following the classical method.

SG of the brain was carried out on a gamma camera (Ohio Nuclear, U.S.) following the classical method in dynamic and static modes using the TC 99M pertechnet at 555 .
REG was conducted by means of "Reospektr-8" (Neurosoft, Russia) in accordance with the standard automated method determining pulse volume disorders in the hemispheres of the brain.

MUGA of the brain was performed on apparatus Advantx (GE) following the classical method of transfemoral access. Simultaneously, taking into account the start and the speed of the injection, $10-12 \mathrm{ml}$ of Omni-pack 350 was introduced intra-carotidally and $7-8 \mathrm{ml}$ intravertebrally. Registration was carried out in direct and side projections in constant subtraction mode at a speed of 25 frames per second. Further on the angiograms obtained in each phase of contrast enhancement were analysed frame by frame [17]. Capillary density analysis was performed in the appropriate phase with an automatic method by means of the computer program "Angio Vision" based on determining the degree of blackening of the corresponding image section [18].

Laboratory examination was conducted according to generally accepted interventional cardiology schemes and included clinical, biochemical and coagulological examination.

\subsection{Type of Atherosclerotic Lesion}

According to the type of atherosclerotic lesions of brachiocephalic arterial branches, the examined patients were divided in the following way:

- intracranial type of lesion-501 (44.53\%) patients;

- mixed type-519 (46.19\%) patients;

- extracranial type-105 (9.33\%) patients.

\subsection{Selection of Patients}

$\mathrm{VaD}$, along with atherosclerotic lesions of the brain, was identified among 665 (59.11\%) patients. In this group, there were 478 (71.87\%) male patients and 187 (28.13\%) female patients.

Assessment of the severity of dementia was conducted in accordance with J. C. Morris's 1993 classification (The Clinical Dementia Rating) [19].

Assessment of cognitive disorders was carried out by means of the Mini-Mental State Examination (MMSE) [20] (among patients who were physically able to perform the tests).

Assessment of everyday life disorders was done using the Index Bartels Functional Evaluation: The Barthel Index (IB) [21].

Initial testing was performed during a patient's first examination or on the day of his/her hospitalization. Repeated testing was carried out on the day of the patient's discharge, and then at intervals of 6 - 12 months.

According to the severity of dementia, the patients were divided into the following groups:

- Group 1 (severity of dementia corresponds to CDR-1) 
-352 (52.93\%) patients;

- Group 2 (severity of dementia corresponds to CDR-2) -184 (27.67\%) patients;

- Group 3 (severity of dementia corresponds to CDR-3) -129 (19.40\%) patients.

Criteria for the selection of patients allowing for the examination and treatment:

1) Consent of the patient and his or her relatives;

2) The patient's medical condition;

3) The severity of dementia and cognitive impairment, the state of higher mental functions and social adjustment, the severity of intellect disorders;

4) The severity of involutive and vascular changes in the brain.

To perform transcatheter treatment following the method of transluminal laser revascularization, 639 (96.09\%) patients were selected.

The groups consisted of patients without serious comorbidities not allowing for the examination and treatment, with fairly satisfactory medical condition in accordance with their age group.

Identified postischemic changes in the brain were subdivided into:

- general involutive changes of the cerebral cortex accompanied by an extension of the subarachnoid space were detected in 569 (89.05\%) cases;

- expansion of Sylvius fissures was detected in 521 (81.53\%) cases;

- elimination of the boundaries of gray and white matter of the brain was detected in 436 (68.23\%) cases;

- signs of unocclusive hydrocephalus were diagnosed in 365 (57.12\%) cases;

- leucoariosis manifestations were detected in 187 (29.26\%) cases;

- macro- and medium focal postischemic brain cysts were detected in 160 (25.04\%) cases;

- micro-focal postischemic brain cysts were detected in 29 (4.54\%) cases;

- single and multiple microcysts were found in 177 (27.70\%) cases;

- absence of postischemic cysts against the background of atherosclerotic lesions was detected in 273 (42.72\%) cases.

The slowing of blood flow in the cerebral hemispheres to Tmax 10 - $12 \mathrm{sec}, \mathrm{T} 1 / 211$ - $13 \mathrm{sec}$ was observed in 401 (62.75\%) cases; to Tmax 14 - 15 sec, T1/2 15 - 20 sec-in 238 (37.25\%) cases.

Lowering of voluminal pulse blood in the carotid and vertebral systems by $20 \%$ - $40 \%$ was detected in 376 (58.84\%) cases, by 40\% - 60\%-in 263 (41.16\%) cases.

Occlusive or stenotic atherosclerotic lesions in the pool of the front, middle and posterior cerebral arteries, accompanied by the deposition of calcium salts in the walls of blood vessels, were detected among all 639 (100\%) patients of the test group.

\subsection{The Method of Transluminal Laser Revascularization of Cerebral Vessels $[17,18,22]$}

(The efficacy and safety of this method have been proved in earlier experimental and clinical studies [23-26].)

To conduct revascularization of main intracranial arteries high-energy laser systems were used; for revascularization of the distal intracranial branches low-energy laser systems were used $[17,18,26]$.

Under local anesthesia, according to Seldinger's classical method, the common femoral artery is punctured and catheterized by installing an introducer with a diameter of $6 \mathrm{~F}-9 \mathrm{~F}$. Through this introducer and through coaxially brought guiding catheters installed in the general and further on in the internal carotid artery, is brought a flexible fiber-optic laser instrument with a diameter of 50 to 200 microns, coupled with a laser unit. The fiber-optic instrument is guided to the site of intracerebral arteries lesion, after which the laser treatment is carried out. The distal end of the fiber-optic instrument is constantly washed with heparinized saline solution. To carry out $\mathrm{X}$-ray control, small doses of radiopaque substance are periodically introduced. The exposure time depends on the nature of the atherosclerotic lesion, the type of the laser system used, and takes from 30 seconds to $40 \mathrm{~min}-$ utes. After the endovascular intervention the patient undergoes repeated cerebral multi-gated angiography following the procedure described above, the results of which determine the degree of revascularization and restoration of the cerebral vascular bed $[18,26]$. We assume that if the capillary blood flow is not fully restored during the first attempt of the intervention, the manipulation can be repeated, but it was not necessary during the transcatheter interventions described.

Postoperative treatment was carried out by common interventional angiocardiology schemes and dosing. The patients underwent conventional desagrigant, anticoagulant and vasodilator therapy including Aspirin, Heparin, and indirect anticoagulants (depending on the blood coagulation indicators), Pentoxifylline $100 \mathrm{mg}$, Complamin 150 mg, Inosin 200 mg, Nootropil (Piracetam) 1200 mg (or Gliatilin $1000 \mathrm{mg}$ ) intravenously, drop by drop, No. 10 - 15; and then they took tablets. Subsequent 3-months courses of tablets were repeated twice a year.

\section{RESULTS}

\subsection{Preliminary Analysis}

It should be noted that among patients who have had ischemic stroke, the severity of dementia is not always directly related to the size of the ischemic focus and is often associated with impaired hemodynamics and seve- 
rity of stenotic lesions of the arterial branches of other pools.

The clinical outcome depended on the severity of the ischemic lesion, the severity of the initial dementia, the expansion of atherosclerotic lesions of cerebral vessels and the timing of transcatheter intervention after the development of the disease symptoms.

\subsection{Treatment Examples}

Here are some examples.

Patient S., male, 57 years old. Suffered a massive stroke of the right hemisphere. Severe vascular dementia, CDR-3. 12 months after the stroke CT of the brain revealed an extensive postischemic cyst in the right hemisphere (Figure 1) sized $7.5 \times 4.8 \times 6.2 \mathrm{~cm}$. SG and REG showed a marked reduction of blood flow and pulse blood with predominance of the process in the right.

Cerebral MUGA revealed subtotal stenosis of the distal part of the trunk of the right internal carotid artery (Figure 2).

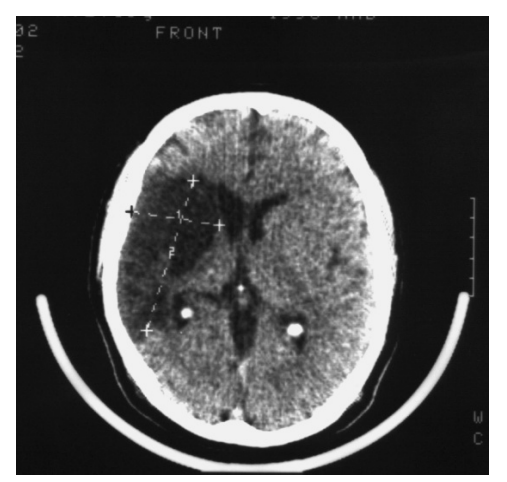

Figure 1. Example: Patient S. male, 57 years old (CDR-3): CT of the brain. BEFORE THE OPERATION: a huge postischemic cyst of $7.5 \times 4.8 \times 6.2 \mathrm{~cm}$ in the right middle cerebral artery region.

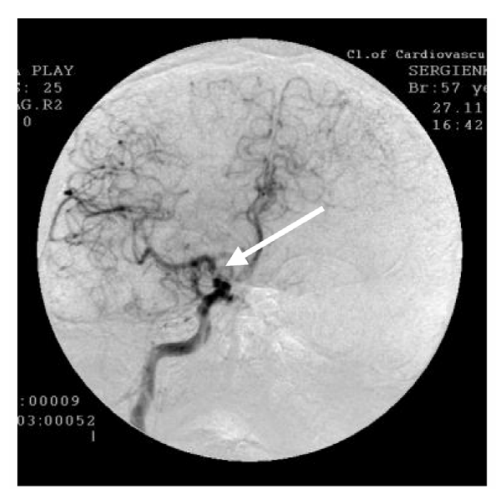

Figure 2. The same patient S.: rightsided carotid MUGA (direct view). BEFORE THE OPERATION: subtotal stenosis of the distal part of the trunk of the right internal carotid artery.
The patient underwent transluminal laser revascularization of the right internal carotid artery. Postoperative MUGA showed completely restored patency and lumen of the distal part of the right internal carotid artery (Figure 3) and pronounced collateral revascularization.

SG and REG revealed positive dynamics of blood flow and pulse blood in the hemispheres of the brain.

In 3 months the patient demonstrated the restoration of motor functions, intellectual abilities and professional skills. The severity of dementia was reduced to CDR-1, and the patient returned to his work.

12 months after the treatment no dementia symptoms were observed; repeated CT of the brain revealed reduction in the size of the postischemic cyst (Figure 4).

5 years after the transcatheter treatment CT (Figure 5) showed a further significant reduction in the size of the postischemic cyst.

Cerebral MUGA (Figure 6) revealed completely pre-

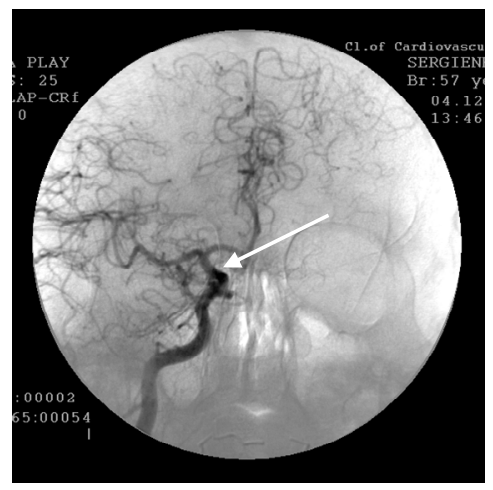

Figure 3. The same patient S.: rightsided carotid MUGA (direct view). AFTER THE OPERATION: complete restoration of permeability and lumen of the trunk of the right internal carotid artery.

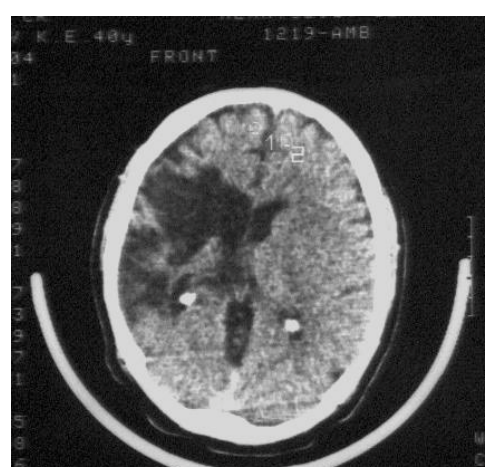

Figure 4. The same patient S.: CT of the brain 12 MONTHS AFTER LASER REVASCULARIZATION: significant decrease in the size of the postischemic cyst with the sings of brain tissue regeneration. There are no symptoms of dementia. 


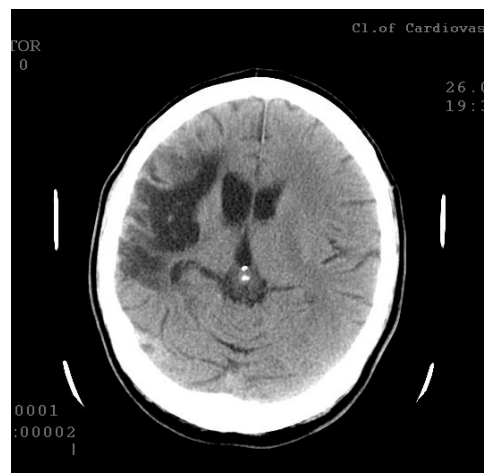

Figure 5. The same patient S.: CT of the brain 5 YEARS AFTER THE TREATMENT: further reduction of the postischemic cyst, progression of regenerative changes. There are no symptoms of dementia.

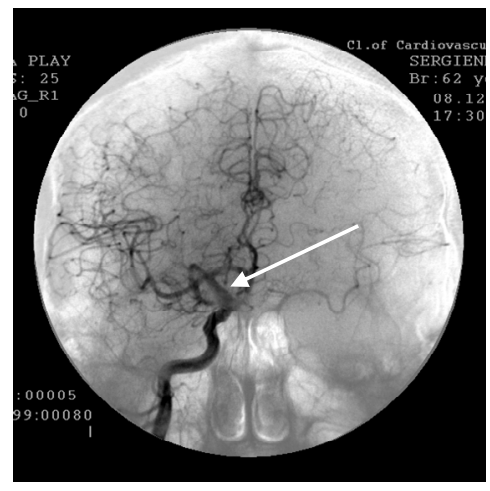

Figure 6. The same patient S.: rightsided carotid MUGA (direct view). 5 YEARS AFTER THE OPERATION: permeability and lumen of the middle cerebral artery trunk completely preserved, further progression of collateral revascularization.

served patency and lumen of the right internal carotid artery and further progression of collateral revascularization. Clinically, there were no signs of dementia.

Patient A., female, 58 years old. Suffered a massive stroke of the right hemisphere. Vascular dementia, CDR-2. 8 months after the disease cerebral MRI revealed an extensive postischemic cyst in the occipital-parietal region of the right hemisphere (Figure 7).

SG and REG showed a marked reduction of blood flow and pulse blood with predominance of the process in the right.

Cerebral MUGA revealed occlusions of the distal branches of the right middle cerebral artery (Figure 8).

The patient underwent transluminal laser revascularization. Postoperative cerebral MUGA (Figure 9) showed a complete restoration of the distal branches of right middle cerebral artery and pronounced collateral revascularization.

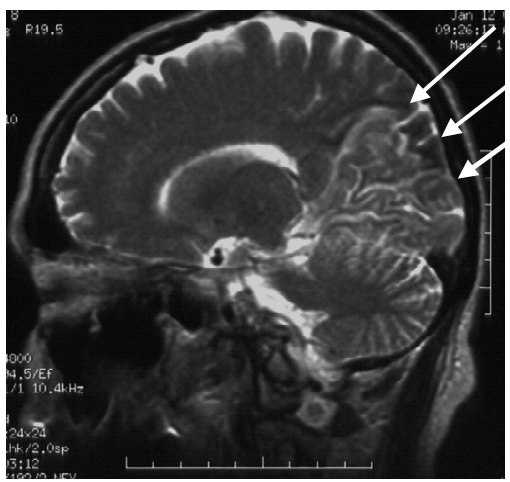

Figure 7. Example: Patient A., female, 58 years old (CDR-2): MRI of the brain. BEFORE THE OPERATION: a huge postishemic cyst in the right occipitoparietal region of the right hemisphere.

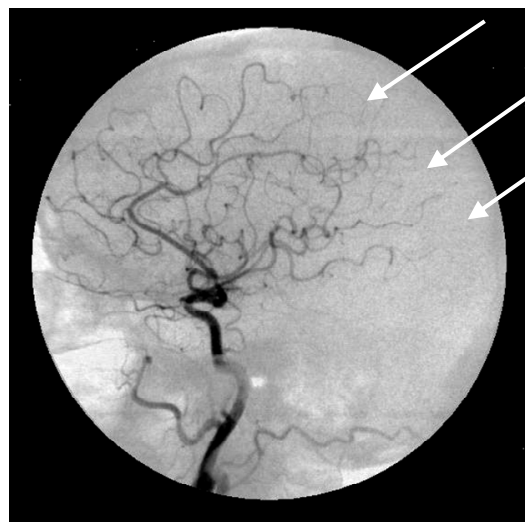

Figure 8. Same Patient A.: right-sided carotid MUGA (lateral projection) BEFORE THE OPERATION: occlusion of distal branches of the right middle cerebral artery.

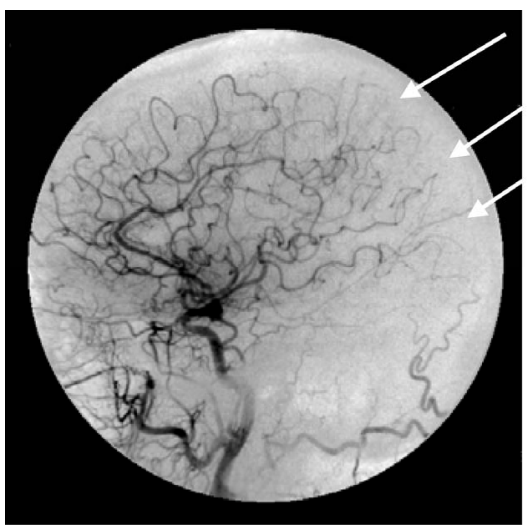

Figure 9. Same Patient A.: right-sided carotid MUGA (lateral projection). AFTER THE OPERATION: restoration of the lumen and permeability of distal branches of the right middle cerebral artery, improvement of collateral blood flow. 
SG and REG showed positive dynamics of blood flow and pulse blood in the hemispheres of the brain. In 3 months the patient demonstrated the improvement of motor functions and intellectual ability; the severity of dementia was reduced to CDR-1.

10 months after the endovascular treatment, repeated cerebral MRI (Figure 10) revealed a significant decrease in the size of the postischemic cyst with elements of brain tissue structure restoration. Clinically, there were no signs of dementia.

Patient K, male, 38 years old. Complaints about memory loss, communication difficulties, frequent impossibility to concentrate, fatigue, vestibular disorders. SG and REG showed the reduction of blood flow and pulse blood on both sides. Clinically, the severity of dementia equals CDR- 1 .

CT of the brain (Figure 11) showed signs of involutive changes and incipient atrophy of the cerebral cortex,

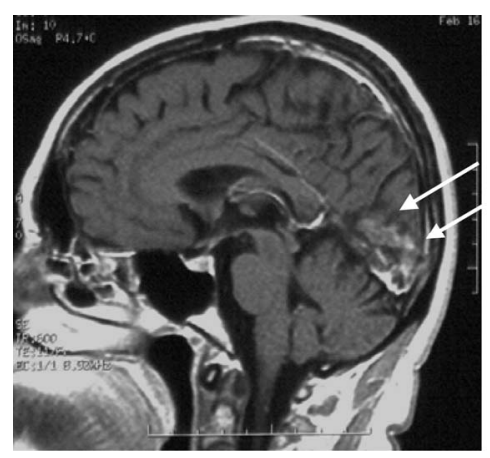

Figure 10. Same Patient A.: MRI of the brain. 10 MONTHS AFTER THE OPERATION: significant decrease in the size of the postischemic cyst with the signs of brain tissue structure reconstruction. There are no symptoms of dementia.

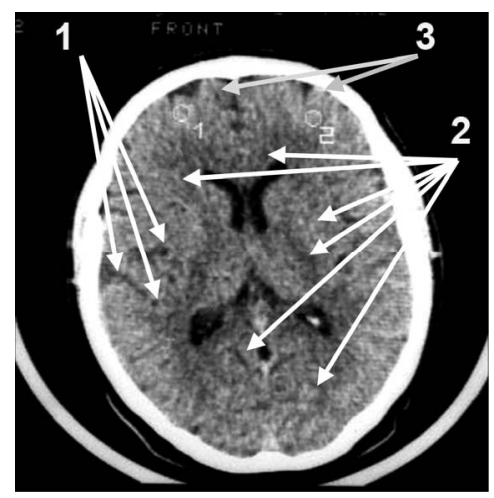

Figure 11. Example: Patient K., male, 38 years old (CDR-1): CT of the brain. BEFORE THE OPERATION: 1. Multiple postischemic cysts; 2. Increase of calcium in the vascular wall; 3. Symptoms of atrophic changes. multiple postischemic microcysts, calcium salts deposits in the walls of blood vessels.

Cerebral MUGA revealed multiple stenoses of the distal branches of the left and right middle cerebral arteries (Figure 12).

The patient underwent transluminal laser revascularization of the distal parts of the right and left middle cerebral arteries. The postoperative cerebral MUGA (Figure 13) showed restoration of the distal branches of the right and left middle cerebral arteries and a pronounced collateral revascularization.

SG and REG demonstrated positive dynamics of blood flow and pulse blood in the hemispheres of the brain.

The treatment caused the disease symptoms to rapidly regress, the patient's complaints stopped and he went back to his daily work; no relapse was observed.

8 years after the endovascular treatment, repeated cerebral CT (Figure 14) showed restoration of the patient's brain tissue structure.

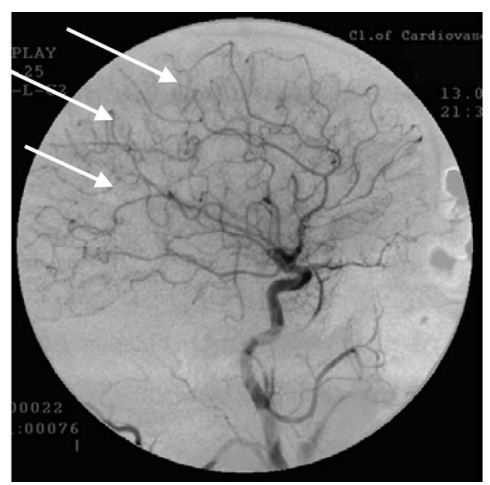

Figure 12. Same Patient K.: leftsided carotid MUGA (lateral projection). BEFORE THE OPERATION: multiple stenosis of the distal branches of the left middle cerebral artery.

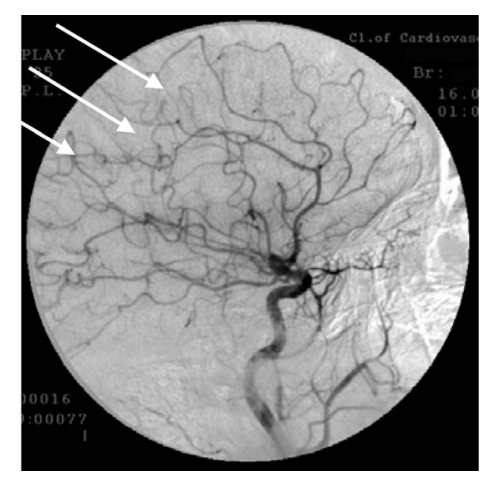

Figure 13. Same Patient K.: leftsided carotid MUGA (lateral projecttion). AFTER THE OPERATION: restoration of the lumen and permeability of distal branches of the left middle cerebral artery, improvement of collateral blood flow. 


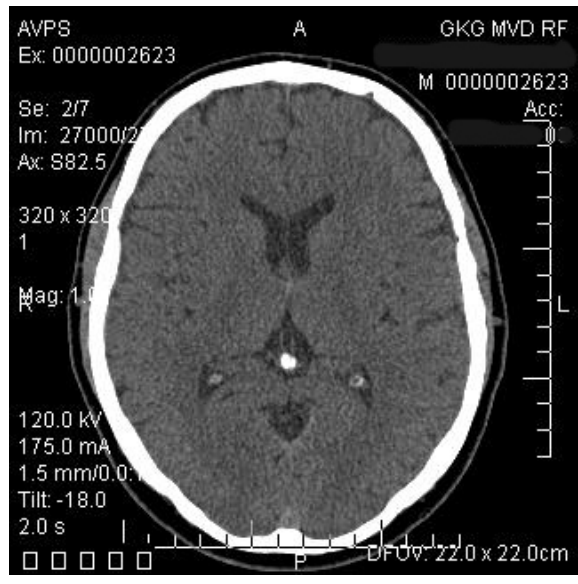

Figure 14. Same Patient K.: CT of the brain. 8 YEARS AFTER TWO-SIDED TRANSLUMINAL LASER REVASCULARIZATION: Restoration of brain tissue structure. There are no symptoms of dementia.

\subsection{Final Analysis}

The treatment carried out has improved the patients' condition, reduced their level of dementia and cognitive disorders, and has allowed shifting them into an earlier CDR-group, or transferring them to the group of patients without dementia.

- Good long-term clinical outcome after the transcatheter laser treatment: almost complete recovery of motor functions and intellectual ability, reduction of cognitive impairment to MMSE 27 - 30, restoration of daily activities to IB $90-100$.

- Satisfactory clinical outcome: incomplete recovery of motor functions and intellectual ability, reduction of cognitive impairment to MMSE 21 - 26, restoration of daily activities to IB 75 - 85 .

- Relatively satisfactory clinical outcome: partial recovery of motor functions and intellectual ability, reduction of cognitive impairment to MMSE 17 - 20, restoration of daily activities to IB 60 - 70 .

In 12 months, various groups of the treated patients demonstrated the following clinical outcome:

- Initial Group 1(severity of dementia corresponds to CDR-1): good clinical outcome was obtained in 282 (80.11\%) cases, satisfactory clinical outcome-in 53 $(15.06 \%)$ cases, relatively satisfactory clinical outcome-in 17 (4.83\%) cases.

- Initial Group 2 (severity of dementia corresponds to CDR-2): good clinical outcome was obtained in 81 (44.02\%) cases, satisfactory clinical outcome-in 62 $(33.70 \%)$ cases, relatively satisfactory clinical outcome-in 41 (22.28\%) cases.

- Initial Group 3 (severity of dementia corresponds to CDR-3): good clinical outcome was obtained in 9 $(8.73 \%)$ cases, satisfactory clinical outcome-in 31
(30.09\%) cases, relatively satisfactory clinical outcome-in 63 (61.16\%) cases.

\subsection{Statistical Analysis}

Statistical analysis of the data is presented in Table $\mathbf{1}$.

Data processing was carried out using the software package Statistica 10 of the Statsoft Company. The analysis of the contingency table comprised according to clinical data was based on the chi-square test (the Pearson Chi-square $=234.95, \mathrm{p}<0.0001$; the resulting Chisquare value of 234.95 is significant beyond the 0.001 level) and showed that the null hypothesis of the independence of the studied traits is rejected, i.e. there is a statistically significant relationship between belonging to the group and the clinical outcome.

It should be noted that among patients who had had an ischemic stroke the restoration of motor functions went on slower than the restoration of intellectual ability and cognitive functions and to a greater degree depended on the size of the postischemic cyst, the timing of transcatheter treatment after the stroke and the severity of motor disorders.

\subsection{Complications}

In carrying out the transcatheter treatment there was no thrombosis or embolism in the distal arterial bed. There were no negative effects after the transluminal laser revascularization. After the intervention, 2 patients had developed a minor hematoma at the puncture site of the femoral artery which did not require surgical treatment, but it was not a complication of the applied method of treatment.

\section{DISCUSSION}

According to many authors, $\mathrm{VaD}$ mostly develops against the background of intracranial or mixed types of atherosclerotic lesions of cerebral vessels [26,27]. Extracranial lesions of the brachiocephalic branches do not always lead to severe brain ischemia and do not always contribute to the development of cognitive disorders and dementia. Some groups of patients feature occlusions of two extracranial branches which lead to the development of natural collateral blood supply and therefore are virtually asymptomatic, and as a result, the patients are for a long time in a fairly good condition [16,26]. Lesions of this kind are amenable to easy and successful surgical and interventional therapies $[15,16]$, but the restoration of blood flow through the main arteries of the neck does not always solve the problem of developed $\mathrm{VaD}$.

Therapeutic treatment of atherosclerotic lesions of brain vessels, complicated by the development of $\mathrm{VaD}$, it is only effective in the early stages of the disease. Traditional reconstructive surgery and stenting of intracranial 
Table 1. Contingency table for statistical features and their significance.

\begin{tabular}{|c|c|c|c|c|}
\hline \multicolumn{5}{|c|}{ 2-Way Summary Table: Observed Freguencies } \\
\hline & Clinical & Clinical & Clinical & Row \\
\hline Group & $\begin{array}{c}\text { Outcome } \\
\text { Relatively } \\
\text { Satisfactory }\end{array}$ & $\begin{array}{c}\text { Outcome } \\
\text { Satisfactory }\end{array}$ & $\begin{array}{c}\text { Outcome } \\
\text { Good }\end{array}$ & Totals \\
\hline CDR-1 & 17 & 53 & 282 & 352 \\
\hline Column \% & $14.05 \%$ & $36.30 \%$ & $75.81 \%$ & \\
\hline Row \% & $4.83 \%$ & $15.06 \%$ & $80.11 \%$ & \\
\hline Total \% & $2.66 \%$ & $8.29 \%$ & $44.11 \%$ & $55.09 \%$ \\
\hline CDR-2 & 41 & 62 & 81 & 184 \\
\hline Column \% & $33.88 \%$ & $42.47 \%$ & $21.77 \%$ & \\
\hline Row \% & $22.28 \%$ & $33.70 \%$ & $44.02 \%$ & \\
\hline Total \% & $6.42 \%$ & $9.70 \%$ & $12.68 \%$ & $28.79 \%$ \\
\hline CDR-3 & 63 & 31 & 9 & 103 \\
\hline Column \% & $52.07 \%$ & $21.23 \%$ & $2.42 \%$ & \\
\hline Row \% & $61.17 \%$ & $30.10 \%$ & $8.74 \%$ & \\
\hline Total \% & $9.86 \%$ & $4.85 \%$ & $1.41 \%$ & $16.12 \%$ \\
\hline Totals & 121 & 146 & 372 & 639 \\
\hline Total \% & $18.94 \%$ & $22.85 \%$ & $58.22 \%$ & $100.00 \%$ \\
\hline \multicolumn{5}{|c|}{ Statistics: Group (3) × Clinical Outcome (3) } \\
\hline Statistic & Chi-square & df & $\mathrm{p}$ & \\
\hline Pearson Chi-square & 234.9514 & $\mathrm{df}=4$ & $p=0.0000$ & \\
\hline M-L Chi-square & 236.3459 & $\mathrm{df}=4$ & $\mathrm{p}=0.0000$ & \\
\hline
\end{tabular}

branches is very difficult due to the vessels small diameter and their complex anatomical location $[15,16]$. Nowadays the restoration of intracranial branches of a small diameter, as well as the stimulation of the collateral and, moreover, the capillary system can be managed only by transcatheter methods using laser energy and fiber optic instruments of very small diameters $[18,26]$.

Given our experience in applying these techniques [23, $24,26]$, it can be concluded that the proposed method is sufficiently safe and efficient. This method is less dangerous than the common technique of transcatheter closure of intracranial vessels aneurysms [28].

Brain tissue has a low degree of absorption of the visible spectrum laser energy, and therefore, the depth of penetration is a few centimeters [26]. As a result, we cannot exclude that laser treatment effects not only the atherosclerotic tissue and the vascular wall causing the restoration of the arterial and the development of the collateral and capillary blood flow [17,18,26,29], but it also effects the brain tissue itself. It is likely that transmission of laser energy stimulates metabolic processes in neurons $[17,18]$. It is possible that subsequent increase of brain tissue mass indicates the development of various regenerative processes $[3,26,30]$. It is confirmed by a growing number of studies which prove the possibility of regenerative processes in unaffected and ischemic cere- bral tissue, both in the experiment and in clinical practice [31,32].

Thus, it can be concluded that vascular dementia mostly develops against the background of distal lesions of intracranial arterial branches.

The method of transluminal laser revascularization of cerebral vessels is an effective transcatheter method of small traumacy for the treatment of arterial lesions of the brain complicated by VaD development. The method allows restoring the patency and lumen of vessels of various diameters simultaneously causing collateral revascularization of the ischemic area and near located tissue which in turn reduces the level of dementia and allows patients to be transferred to an earlier CDR-stage, or even, after the complete regression of dementia, to go beyond this scale. The effect persists for a long time; it promotes further regression of $\mathrm{VaD}$ signs and improves the patients' quality of life.

\section{REFERENCES}

[1] Gillum, R.F., Kwagyan, J. and Obisesan, Th.O. (2011) Ethnic and geographic variation in stroke mortality trends. Stroke, 42, 3294-3296. doi:10.1161/STROKEAHA.111.625343

[2] Frölich, A.M., Psychogios, N.M., Klotz, E., Knauth, M. 
and Knauth, P. (2012) Angiographic reconstructions from whole-brain perfusion CT for the detection of large vessel occlusion in acute stroke. Stroke, 43, 97-102. http://stroke.ahajournals.org/content/43/1/97.abstract doi:10.1161/STROKEAHA.111.630954

[3] Zhulev, N.M., Pustozertsev, V.G. and Zhulev, S.N. (2002) Cerebrovascular diseases. Biological Network Manager (BINOM), Moscow. http://www.combook.ru/product/158808/

[4] Pendlebury, S.T., Wadling, S., Silver, L.E. and Rothwell, P.M. (2011) Transient cognitive impairment in TIA and minor stroke. Stroke, 42, 3116-3121. doi:10.1161/STROKEAHA.111.621490

[5] Skoog, I. (2011) Psychiatric disorders in the elderly. Canadian Journal of Psychiatry, 57, 387-397. http://www.ncbi.nlm.nih.gov/pubmed/21835102

[6] Kalaria, R.A., Kenny, R.A., Ballard, C.G., Perry, R., Ince, P. and Polvikoski, T. (2004) Towards defining the neuropathological substrates of vascular dementia. Journal of the Neurological Sciences, 226, 75-80. doi:10.1016/j.jns.2004.09.019

[7] Kalaria, R.N. (2010) Vascular basis for brain degeneration: Faltering controls and risk factors for dementia. $\mathrm{Nu}$ trition Reviews, 68, S74-S87. doi:10.1111/j.1753-4887.2010.00352.x

[8] Pasi, M., Poggesi, A., Salvadori, E. and Pantoni, L. (2012) Post-stroke dementia and cognitive impairment. Front Neurol Neurosciences, 30, 65-69. doi:10.1159/000333412

[9] ElAli, A., Doeppner, T.R. and Hermann, D.M. (2011) Increased blood-brain barrier permeability and brain edema after focal cerebral ischemia induced by hyperlipidemia: Role of Lipid peroxidation and calpain-1/2, matrix metalloproteinase-2/9, and RhoA overactivation. Stroke, 42, 3238-3244. doi:10.1161/STROKEAHA.111.615559

[10] Moustafa, R.R., Momjian-Mayor, I., Simon Jones, P., Morbelli, S., Day, D.J., Aigbirhio, F.I., Fryer, T.D., Warburton, E.A. and Baron, J.C. (2011) Microembolism versus hemodynamic impairment in rosary-like deep watershed infarcts: A Combined positron emission tomography and transcranial doppler study. Stroke, 42, 31383143. doi:10.1161/STROKEAHA.111.616334

[11] Roman, G.C. (2004) Facts, myths, and controversies in vascular dementia. Journal of the Neurological Sciences, 226, 49-52. doi:10.1016/j.jns.2004.09.011

[12] Kavirajan, H. and Schneider, L.S. (2007) Efficacy and adverse effects of cholinesterase inhibitors and memantine in vascular dementia: A meta-analysis of randomised controlled trials. The Lancet Neurology, 6, 782-792. doi:10.1016/S1474-4422(07)70195-3

[13] Roman, G.C. and Kalaria, R.N. (2006) Vascular determinants of cholinergic deficits in Alzheimer disease and vascular dementia. Neurobiology of Aging, 27, 1769-1785. doi:10.1016/j.neurobiolaging.2005.10.004

[14] Liu, R., Yuan, H., Yuan, F. and Yang, S.H. (2012) Neuroprotection targeting ischemic penumbra and beyond for the treatment of ischemic stroke. Journal of Neurology
Research, 34, 331-337.

http://www.ncbi.nlm.nih.gov/pubmed/22643076

[15] Silver, F.L., Mackey,A., Clark,W.M., Brooks, W., Timaran, C.H., Chiu, D., Goldstein, L.D., Meschia, J. F., Ferguson, R.D., Moore, W.S., Howard, G. and Brott, T.G. (2011) Safety of stenting and endarterectomy by symptomatic status in the Carotid Revascularization Endarterectomy versus Stenting Trial (CREST). Stroke, 42, 675680. doi:10.1161/STROKEAHA.110.610212

[16] Papanagiotou, P., Roth, C., Walter, S., Behnke, S., Grunwald, L.Q., Viera, J., Politi, M., Körner, H., Kostopoulos, P., Haass, A., Fassbender, K. and Reith, W. (2011) Carotid artery stenting in acute stroke. Journal of the American College of Cardiology, 58, 2363-2369. doi:10.1016/j.jacc.2011.08.044

[17] Maksimovich, I.V. (2006) Method for carrying out transluminal laser-induced brain revascularization in atherosclerotic injury cases. RF Patent No. 2297861.

http://worldwide.espacenet.com/publicationDetails/biblio ?II $=3 \& N D=3 \&$ adjacent $=$ true\&locale $=$ en_EP\&FT $=$ D\&dat $\mathrm{e}=20070427 \& C C=\mathrm{RU} \& N R=2297861 \mathrm{C} 1 \& \mathrm{KC}=\mathrm{C} 1$

[18] Maksimovich, I.V. (2006) Method of transluminal laser revascularization of cerebral blood vessels having atherosclerotic lesions. US Patent No. 7490612.

http://www.patentlens.net/patentlens/patents.html?patnu ms=US_7490612\&returnTo=patentnumber.html\%3Fquer y\%3D\%2528US_7490612\%2Bin\%2Bpublication_numbe r\%2529

[19] Morris, J.C. (1993) The clinical dementia rating (CDR): Current version and scoring rules. Neurology, 11, 24122414. doi:10.1212/WNL.43.11.2412-a

[20] Folstein, M.F., Folstein, S.E and McHugh, P.R. (1975) Mini-mental state, a practical method for grading the cognitive state of patients for the clinician. Journal of Psychiatric Research, 12, 189-198. doi:10.1016/0022-3956(75)90026-6

[21] Mahoney, F.I. and Barthel, D.M. (1965) Functional evaluation: The barthel index. Maryland State Medical Journal, 14, 61-65.

http://www.ncbi.nlm.nih.gov/pubmed/14258950

[22] Maksimovich, I.V. (2010) Transluminal Laser revascularization of cerebral blood vessels in the treatment of ischemic stroke. Journal of the American College of Cardiology, 56, B48-B49. doi:10.1016/j.jacc.2010.08.228

[23] Deviatkov, N.D., Rabkin, I.K.H., Maksimovich, I.V., Rogov, R.A. and Aleinikov, V.S. (1986) Use of copper-vapor laser radiation for the evaporation of atherosclerotic lesions of the major arteries in vitro. Surgery, 4, 116-121. http://www.ncbi.nlm.nih.gov/pubmed/3713043

[24] Petrovskiy, B.V., Deviatkov, N.D., Rabkin, I.K.H., Maksimovich, I.V. and Rogov, K.A. (1986) Destruction of arteriosclerotic lesions of human cadaveric arteries by laser irradiation with copper vapors. Surgery, 5, 112-116. http://www.ncbi.nlm.nih.gov/pubmed/3723942

[25] Kuleshov, E.V. and Maksimovich, I.V. (1994) Endovascular surgery in patients over 65 with disseminated atherosclerosis of the vessels of the pelvis and lower ex- 
tremities. Bulletin of Surgery Named after Grekov, 152, 27-30.

http://www.ncbi.nlm.nih.gov/pubmed/7701734

[26] Maksimovich, I.V. (2004) Transljuminal laser angioplasty in treatment of ischemic lesions of a brain. M.D. dissertation. Russian University of Friendship of the People, Moscow.

http://disseng.com/page/order/id/206426.html

[27] Deramecourt, V., Slade, J.Y., Oakley, A., Perry, R.H., Ince, P.G., Maurage, C.A. and Kalaria, R.N. (2012) Staging and natural history of cerebrovascular pathology in dementia. Neurology, 78, 1043-1050. doi:10.1212/WNL.0b013e31824e8e7f

[28] Piotin, M., Spelle, L., Mounayer, C., Loureiros, C., Ghorbani, A. and Moret, J. (2009) Intracranial aneurysms coiling with matrix: Immediate results in 152 patients and midterm anatomic follow-up from 115 patients. Stroke, 40, 321-323.

http://stroke.ahajournals.org/content/40/1/321.abstract doi:10.1161/STROKEAHA.108.520866

[29] Starck, T., Nissilä, J., Aunio, A., Abou-Elseoud, A., Remes, J., Nikkinen, J., Timonen, M., Takala, T., Tervonen, O. and Kiviniemi, V. (2012) Stimulating brain tis- sue with bright light alters functional connectivity in brain at the resting state. World Journal of Neuroscience, 2, 81-90. doi:10.4236/wjns.2012.22012

[30] Kozlov, V.I. and Azizov, G.A. (2007) Pathophysiological characteristics of microcirculatory disorders in chronic arterial ischaemia of lower limbs. Angiol Sosud Khir, 13, 17-23.

http://www.ncbi.nlm.nih.gov/pubmed/17679971

[31] Heinrich, C., Blum, R., Gascón, S., et al. (2010) Directing astroglia from the cerebral cortex into subtype specific functional neurons. PLoS Biology, 8, e1000373. http://www.ncbi.nlm.nih.gov/pubmed/20502524

[32] Ekonomou, A., Johnson, M., Perry, R.H., Perry, E.K., Kalaria, R.N., Minger, S.L. and Ballard, C.G. (2012) Increased neural progenitors in individuals with cerebral small vessel disease. Neuropathology and Applied Neurobiology, 38, 344-353.

http://www.ncbi.nlm.nih.gov/pubmed/21988073 doi:10.1111/j.1365-2990.2011.01224.x 